\title{
Coopérer pour se découvrir et développer ses compétences sociales
}

\author{
MÉLANIE SOUHAIT \\ IDEFI TalentCampus, Fondation de Coopération Scientifique Bourgogne Franche-Comté, \\ Dijon, melanie.souhait@talent-campus.fr \\ PAULINE THEVENOT \\ IDEFI REMIS, Université de Lorraine, pauline.thevenot@yahoo.com \\ LUCILE HERNANDEZ
}

CIRAP (Centre Interdisciplinaire de Recherches Appliquées au Champ Pénitentiaire), Agen, hernandezlucie@yahoo.fr

SÉBASTIEN CHEVALIER

IDEFI TalentCampus, Fondation de Coopération Scientifique Bourgogne Franche-Comté, Dijon, sebastien.chevalier@u-bourgogne.fr

RAPHAËL E. DUVAL

IDEFI REMIS, Université de Lorraine, raphael.duval@univ-lorraine.fr

\section{Résumé}

Les compétences sociales se travaillent tout au long de la vie et doivent s'apprendre au plus tôt car elles sont indispensables dans la vie professionnelle. La coopération notamment est une compétence essentielle dans une entreprise et c'est pour cela qu'elle est utilisée comme moyen pédagogique dans la formation proposée par les deux programmes Initiatives d'Excellences en Formations Innovantes (IDEFI). Ceux-ci se sont associés pour proposer aux étudiants en L2 Sciences pour la Santé de l'Université de Lorraine de «booster leur réussite» par le développement des compétences sociales. Grâce à une semaine de formation basée sur la coopération, les étudiants ont pu travailler leurs compétences sociales et augmenter leur confiance en eux.

\begin{abstract}
Soft skills have to be trained throughout life and must be learned as early as possible because they are essential in professional life. Cooperation is particularly useful in a compagny and that is why it is used as a teaching aid in the training created by the two Innovative Training
\end{abstract}


Initiatives programs. They worked together to offer L2 Sciences for Health students at Lorraine University to "boost their success" through soft skills development. Thanks to a week of training based on cooperation, the students were able to work on their social skills and increase their self-confidence.

\section{Mots-clés}

Compétences sociales, travail en équipe, communication, coopération, évaluation de la formation

\section{Key words}

Soft skills, teamwork, communication, cooperation, training assessment

\section{Introduction}

Ce texte a pour objectif de présenter une expérimentation qui s'est déroulée à l'Université de Lorraine, fruit d'une coopération entre les projets IDEFI TalentCampus (ANR-11-IDEFI-0035) et REMIS (ANR-11-IDEFI-0033). Ce dispositif d'une semaine vise notamment, par le travail en groupe, à faire prendre conscience aux étudiants de leurs compétences sociales clés afin qu'ils puissent les développer et les valoriser notamment devant un recruteur. En effet, à diplôme égal, deux étudiants postulant pour un même travail, devront prouver leur «plusvalue » en valorisant les compétences développées depuis leur plus jeune âge, dans leur scolarité, leur enfance, leurs études ou encore leurs activités extra-scolaires. Nous appelons cela les compétences sociales. Celles-ci représentent un processus continu et dynamique, qui résulte d'une trajectoire individuelle et sociale et se construit de manière plus ou moins régulière, sous l'influence de différents facteurs, tout au long de la vie. Les compétences sociales permettent notamment à l'individu d'être « socialement efficace », c'est-à-dire de créer et de maintenir des relations sociales adéquates et de «qualité » (Drozda-Senkowska et coll., 2002 ; Giret et Morlaix, 2016). Dans cet article, nous nous intéressons particulièrement à la notion de coopération (Goleman, 1999) car c'est une compétence sociale souvent requise dans le monde professionnel pour favoriser le travail d'équipe et l'intelligence collective, mais aussi dans la vie quotidienne (sports d'équipe, activités collectives en famille ou entre amis). L'objet de cette publication est alors d'étudier ce que l'expérimentation mise en place par les deux projets IDEFI a permis aux étudiants de développer et d'acquérir grâce à un travail basé sur la coopération : ont-ils réellement développé leurs compétences sociales? Ont-ils créé une cohésion de 
promotion ? Ont-ils acquis des techniques de travail en groupe et de communication interpersonnelle? Quels bénéfices retirent-ils de cette semaine basée sur l'échange et le relationnel ?

\section{Cadre théorique}

Le rapport de 1'OCDE publié en 2015 et intitulé «Skills for Social Progress: The Power of Social and Emotional Skills » témoigne de l'importance de développer les compétences sociales grâce à l'éducation et à la formation. En effet, elles peuvent avoir des répercussions aussi bien sur la vie personnelle et la santé mentale que sur la réussite et les résultats sociaux, professionnels ou scolaires (OCDE, 2015 ; Moscovici et coll., 2005).

Mais que sont les compétences sociales ? Cette question fait l'objet de nombreux débats dans la littérature quant à sa définition et sa mesure. En effet, en fonction de la position disciplinaire, théorique et épistémologique adoptée, les compétences sociales n'auront pas le même sens et regrouperont des réalités variées (Hernandez, 2016). Cette abondante littérature témoigne de la complexité de cette notion, et ainsi, de la difficulté à la cerner. Dans sa plus simple acception, les compétences sociales sont considérées comme des « habiletés » permettant aux individus d'adopter des attitudes et des comportements acceptables, c'est-à-dire adaptés et attendus par rapport aux normes sociales de notre société (Segrin, 2000 ; Spence, 2003). La notion de compétences sociales implique cependant bien d'autres aspects. À partir de travaux récents sur le sujet, nous avons dégagé les éléments clés permettant de définir ce que sont les compétences sociales (Abbet et Moreau, 2012 ; Coulet, 2011 ; Drozda-Senkowska et coll., 2002 ; Nangle et coll., 2010). Nous présentons ci-dessous quelques éléments communs à la majorité de ces travaux, qui, même s'ils ne font pas l'objet de consensus, nous paraissent relativement récurrents :

- Les compétences sociales représentent un processus continu et dynamique. Ce processus résulte d'une trajectoire individuelle et sociale et se construit de manière plus ou moins régulière tout au long de la vie, sous l'influence de processus développementaux, sociaux, affectifs, cognitifs ou encore contextuels.

- Elles permettent à l'individu d'être « socialement efficace », c'est-à-dire de créer et de maintenir des relations sociales adéquates et de « qualité ».

- Les compétences sociales se recoupent selon deux dimensions complémentaires et interdépendantes : une dimension intrapersonnelle (qui relève de la gestion de soi) et 
une dimension interpersonnelle (qui relève de la gestion des interactions avec l'environnement social) ;

Au regard de l'importance des problèmes actuels sur le marché de l'emploi d'un côté, et du rôle favorable et complémentaire des compétences sociales sur l'insertion sociale et professionnelle d'un autre côté, on constate une importante volonté de développer ces compétences, que ce soit dans le milieu de l'enseignement supérieur ou de l'entreprise. Dans le contexte scolaire, plusieurs chercheurs soulignent qu'un climat socio-affectif favorable dans la classe aura des effets positifs et significatifs sur la motivation et l'implication des étudiants (Sarrazin, Tessier et Trouilloud, 2006 ; Duru-Bellat, 2004 ; Charlot, Bautier et Rochex, 1992). Soutenir le développement socio-émotionnel des étudiants leur permet également d'obtenir de meilleurs résultats sur le plan académique et social (Desbiens et coll., 2000), et cela « non seulement dans un contexte scolaire mais également tout au long de leur vie » (Durlak et coll., 2011). Favoriser les compétences sociales des étudiants est par conséquent directement positif pour leur développement social, affectif et cognitif, mais il permet également une aide importante au niveau du travail d'enseignement lié aux apprentissages disciplinaires (Curchod et coll., 2012). L'approche socioconstructiviste (Vygotsky, 1978 ; Wallon, 1941) sur laquelle s'appuie ce travail donne une place centrale aux dimensions sociales et interactionnelles dans le développement des compétences. Pour le dire autrement, nous considérons l'individu comme un être social qui co-construit ses connaissances et développe ses compétences grâce à ses expériences personnelles et aux interactions et confrontations avec son environnement social (Joannaert, 2009 ; Vygotsky, 1978). En ce sens, les compétences représentent un construit socio-culturel. Elles s'élaborent dans un contexte particulier qui lui donne sens. Elles ne peuvent pas être transmises par un simple enseignement décontextualisé et vertical (Joannaert, 2009). L'individu est également actif dans ses apprentissages et sa place, ses représentations, ses expériences, sont prises en considération. La coopération, la communication, et plus largement la capacité à travailler en équipe, sont donc au centre de ce type d'apprentissages. Elles sont par ailleurs considérées comme les compétences sociales les plus valorisées par les employeurs (Baron et Markman, 2000). En effet, les personnes coopératives sont capables de s'adapter à n'importe quelles nouvelles situations sociales (Baron et Markman, 2000). Ces auteurs parlent de « caméléons sociaux » capables de parler de n'importe quel sujet, à n'importe quelle personne et dans n'importe quel contexte.

Nous pouvons citer en exemple une expérimentation basée sur l'approche socioconstructiviste, le « scénario collaboratif » d'Eneau et Simonian (2011), qui s'appuie sur le travail en groupe et 
la formation en ligne pour une population d'adultes en reprise d'études. L'objectif est que ces apprenants développent des compétences transversales, relationnelles et communicatives, (négociation, coordination, argumentation, autonomie...) en réinterrogeant leurs expériences et connaissances antérieures (donner du sens aux apprentissages) et en confrontant ces expériences et leur point de vue à celui des autres membres du groupe. L'originalité de ce projet réside donc dans le fait qu'il ne s'agit pas d'une simple transmission de savoirs par un enseignant mais d'une co-construction des savoirs.

Les compétences sociales et le travail d'équipe sont donc fortement liés et c'est pour cela que la formation présentée dans ce texte a pour enjeu le développement des compétences sociales, par la coopération des étudiants.

\section{Questions de l'étude}

\subsection{Problématique}

La Licence Sciences pour la Santé (SpS) de l’Université de Lorraine (UL) a comme spécificité de commencer directement en deuxième année de licence (L2). Les 20 étudiants et 36 étudiantes accueillis sont donc originaires de filières différentes. En 2018, la L2 SpS comptait 36 étudiants issus de L1 Sciences de la Vie, 15 étudiants issus de Première Année Commune des Études de Santé (PACES), 1 redoublant, 1 étudiant de L1 STAPS, 1 étudiant de $3^{\mathrm{e}}$ année de l'Institut de Formation en Soins Infirmiers (IFSI) et 2 diplômés étrangers.

Ce public dispose de connaissances et de méthodes de travail différentes. Les étudiants qui intègrent la L1 SpS suite à la PACES ont par exemple été confrontés à une forte compétition promue par le système classant du concours de la PACES, concours qui favorise aussi l'assimilation de connaissances « par cœur » et provoque la solitude des étudiants. En outre, les étudiants issus de la PACES intègrent la filière $\mathrm{SpS}$ bien souvent parce qu'ils n'ont pas été suffisamment bien classés. Cette filière constitue alors un choix par défaut qui peut être assimilé à un échec. D'après les enseignants, ce sentiment d'échec aurait un impact sur l'estime de soi des étudiants et sur leur capacité à faire des choix pour la suite de leurs études.

\subsection{Présentation des deux projets IDEFI : REMIS et TalentCampus}

Labellisée en 2012, l'IDEFI REMIS (ANR-11-IDEFI-0033) regroupe 7 Universités (Université de Bretagne Occidentale, Université d'Angers, Université Lille 2, Université de Lorraine, Université d'Orléans, Université de Rouen, Université de Montpellier) et 9 partenaires issus du 
monde socio-économique. Cette IDEFI propose des formations dans les secteurs du Management et de l'Ingénierie de la Santé (MIS), qui débouchent sur de nouveaux métiers de la santé s'adressant à tous les publics. L'objectif est d'accompagner le développement des formations en Management et Ingénierie de la Santé par la mise en place d'outils pédagogiques innovants (serious games, table anatomique virtuelle, simulation grandeur nature, apprentissage par problème, etc.).

Depuis 2012, 1'IDEFI TalentCampus (ANR-11-IDEFI-0035) crée des formations permettant aux lycéens, étudiants, salariés et demandeurs d'emploi, de découvrir et développer leurs compétences sociales: optimiser l'impact de sa communication, améliorer son leadership, augmenter sa confiance et son estime de soi, mieux gérer son stress et ses émotions, ou encore apprendre à travailler en équipe.

En 2017 et 2018, les deux IDEFI ont décidé de collaborer pour créer la formation « Booster sa réussite ». Celle-ci s'est donc déroulée durant deux ans à l'Université de Lorraine au mois de septembre. Après avoir présenté le contenu et les objectifs attendus par cette formation, nous présenterons ici les résultats qualitatifs recueillis auprès des étudiants ayant suivi la formation en septembre 2018.

\section{3. « Booster sa réussite » : une expérience menée à l'Université de Lorraine}

Le dispositif «Booster sa réussite », pour aller dans le sens de l'approche socioconstructiviste, propose une pédagogie expérientielle (Kolb, 1984), active, responsabilisante et coopérative, basée à la fois sur le travail en groupe, la mise en pratique fictive et les échanges. Cette approche permet entre autres à chaque étudiant d'être autonome et responsable dans l'acquisition et la construction de nouveaux apprentissages et de nouvelles compétences (par exemple la prise de décision, l'engagement, ou encore l'esprit d'initiative). Ainsi, les étudiants travaillent en équipes de 5 personnes, durant la semaine de formation. Chaque équipe est renouvelée quotidiennement pour maximiser les rencontres et les échanges. Il s'agit alors de travailler autour de situations «problématiques » qui exigent la confrontation des points de vue et la coopération : se servir de ses propres expériences et de l'autre pour co-construire ses connaissances. On parle d' «apprentissage collaboratif » : l'apprenant utilise les ressources du groupe pour apprendre et développer ses compétences sociales clés. 


\subsection{Présentation de la formation}

L'expérimentation que nous présentons s'est déroulée auprès des 56 étudiants de L2 Sciences pour la Santé en septembre 2018. La formation d'une semaine visait à :

- permettre à chacun de découvrir l'importance des compétences sociales (et donc cibler ses compétences fortes pour pouvoir les valoriser notamment devant un recruteur ou dans un $\mathrm{CV}$ ) ;

- travailler l'estime de soi (et commencer à l'améliorer grâce à des outils simples et pratiques pour permettre aux étudiants de regagner confiance en leurs capacités à réussir leurs études) ;

- développer des compétences de travail en groupe et de communication orale (ces deux compétences étant essentielles dans la vie universitaire d'un étudiant).

Différents modules de formation ont été proposés aux étudiants pendant une semaine en s'appuyant sur le modèle de l'apprentissage expérientiel de Kolb (1984). Ce dernier postule que l'apprentissage englobe quatre éléments : une participation active, une observation réflexive, des expériences basées sur la théorie et l'expérimentation de ces théories. À ce modèle, nous rajoutons une dimension sociale selon nous indispensable. La coopération est ainsi placée au centre de chacune de ces phases afin d'aider les étudiants à tirer des conclusions des expériences vécues ensemble et, sur cette base, co-construire des connaissances. La formation « Booster sa réussite »s'est appuyée sur ces concepts pour s'organiser comme suit :

Jour 1 : la formation débute avec un jeu d'entreprise permettant de briser la glace entre les étudiants et de découvrir la pédagogie active. Le jeu consiste en la création d'une entreprise d'aéronautique en équipe de 5 personnes. Ainsi, les étudiants doivent fabriquer des avions en papier pour les envoyer le plus loin possible sur une piste improvisée. Ce challenge est un prétexte permettant de développer plusieurs concepts pédagogiques et durant lequel tous les apprenants de tous âges se désinhibent rapidement. Grâce à ce jeu, les apprenants découvrent leurs propres compétences sociales clés, à travers les échanges avec leurs coéquipiers. C'est lors de cette première journée que des techniques pour comprendre les mécanismes du travail en groupe sont données. Les étudiants commencent alors à se saisir de l'importance de la concertation en équipe et de l'intelligence collective. Des moments de débriefings sont organisés régulièrement avec les formateurs, afin que les équipes s’interrogent sur leur manière d'interagir, de fonctionner. Tous ces outils seront réutilisés au fil de la semaine dans les autres modules de formation. Durant cette journée, un travail sur son parcours personnel et 
professionnel permet également à chaque étudiant de confronter au regard de ses camarades ses compétences développées au fil de sa vie, et de prendre conscience du caractère particulier de chaque parcours de vie. Enfin, une activité permettant de faire un retour sur les compétences fortes de ses coéquipiers est également proposée durant toute la semaine : les apprenants doivent écrire des compétences sociales perçues chez leurs coéquipiers et glisser ces notes dans les «boîtes aux lettres» des autres apprenants. Ces boîtes sous forme d'enveloppes sont accrochées aux murs de la salle principale de formation durant toute la semaine. Dès la fin de la première journée, les étudiants remplissent donc les boîtes de leurs camarades dans un esprit de bienveillance expliqué à tous le matin même. La découverte des mots dans l'enveloppe se fait ensuite au cas par cas : certains apprenants lisent leurs mots chaque jour, d'autres attendent la fin de la formation.

Jour 2: les étudiants suivent une formation «prise de conscience sur l'estime de soi dont l'objectif est de les amener à porter un regard positif sur soi, notamment en citant des réussites qu'ils se reconnaissent, ou en cernant des compétences sociales qu'ils pensent bien maîtriser. Les apprenants sont sensibilisés également à la gestion des émotions et à la gestion du stress. Pour ce faire, des activités manuelles sont proposées aux étudiants : poterie, collage, photographie. Ces activités ludiques et «décalées » sont encore une fois un prétexte pour amener l'étudiant à sortir du cadre en pensant autrement et en changeant ses habitudes de pensées et de réflexion sur soi. Les deux formateurs sont présents toute la journée pour accompagner les étudiants dans cette réflexion et pour leur permettre de faire le lien entre les activités proposées et leur estime de soi. L'enjeu est d'outiller les étudiants pour qu'ils soient prêts à valoriser leurs compétences (notamment sociales) lors d'un entretien par exemple, et sans être submergés par le stress et leurs émotions.

Jour 3: cette journée est destinée à comprendre l'importance de la communication interpersonnelle au service du travail en équipe (reformulation, cadre de référence, filtres...) et notamment du non-verbal lors d'une prise de parole en public, élément à optimiser pour rendre son discours accrocheur et dynamique. Les étudiants peuvent alors s'entraîner à exprimer des idées à l'oral devant leur équipe de travail selon des critères de forme à respecter. Afin que le travail soit axé sur la forme, les apprenants se sont entraînés à partir de textes préalablement écrits qui parlaient de leur vécu.

Jour 4 : le souhait de l'équipe pédagogique était d'amener les étudiants à réfléchir sur leur métacognition c'est-à-dire la représentation qu'ils ont de leurs connaissances et de la façon dont ils peuvent les construire et les utiliser. «C'est par la médiation cognitive que l'enseignant 
donne à l'élève les moyens d'apprendre et donc les clés pour sa réussite scolaire » (Barth, 1993). Ce module permet aux étudiants d'échanger sur leur manière d'apprendre et d'acquérir des techniques de mémorisation qui leur conviennent le mieux et leur sont nécessaires dans le cadre de leurs études.

Jour 5 : la semaine de formation se termine par une « carte blanche ». La consigne donnée aux étudiants est de restituer, en équipes ou de manière individuelle, la semaine passée. Les étudiants doivent alors faire preuve de créativité pour parler de leur expérience de formation et exprimer ce qu'ils retiennent, ont découvert ou ce qu'ils ont l'intention de mettre en place. En 2018, un groupe d'étudiants a écrit un rap, un autre groupe a écrit et joué une saynète, un groupe encore a fait une présentation orale originale. Cette journée permet de faire un bilan de la formation vécue, et aide à ancrer les outils et techniques apprises durant la semaine.

\section{Méthodologie}

La formation a été évaluée par les étudiants via des questionnaires d'auto-évaluation proposés en ligne sur une plateforme sécurisée, créée spécifiquement pour évaluer les formations TalentCampus. La formation et les questionnaires ont été pensés et conçus en respectant l'alignement constructif de Biggs (2003), qui incite à d'abord fixer des objectifs d'apprentissage (résultats attendus), et à planifier seulement ensuite les activités proposées aux étudiants pour atteindre ces objectifs et les modalités pédagogiques choisies. Ainsi, tous les questionnaires soumis aux étudiants ont été créés pour vérifier l'atteinte des objectifs fixés en amont de la formation (ce que l'on veut que l'étudiant soit capable de faire), et pour connaître le sentiment des étudiants quant aux contenus et modalités pédagogiques.

Deux niveaux ont été pris en compte pour évaluer l'impact et l'efficacité de cette formation «Booster sa réussite ».

Ainsi, le premier niveau concerne la satisfaction des étudiants vis-à-vis de la formation. Ce niveau est également appelé «niveau de réaction ». Cette évaluation est importante à prendre en compte car l'impression globale des sujets à la fin d'une intervention restera présente et aura un impact sur son travail et sur ses futures expériences. Le deuxième niveau évalue les « acquis ». L'objectif est d'avoir un retour des étudiants sur ce qu'ils ont appris et retenu durant la formation et d'avoir un aperçu de l'évolution des compétences sociales ciblées. 
Le questionnaire propose des questions ouvertes afin que chaque étudiant puisse développer librement ses réponses. Plus précisément, ces questions ouvertes s'inscrivent dans plusieurs grandes thématiques :

- l'atteinte des objectifs d'apprentissage fixés pour chaque journée et sur l'ensemble de la formation (par exemple, «Avez-vous découvert 3 compétences sociales fortes chez vous ? » Ou encore, « Savez-vous expliquer en quoi vos compétences sociales sont utiles dans la mise en place de votre projet d'études ou professionnel ?»),

- l'utilisation des acquis de la formation dans les études mais aussi dans la vie privée des étudiants,

- la satisfaction/l'insatisfaction des étudiants quant au contenu de la formation pour pouvoir faire progresser la formation lors d'une prochaine édition,

- les modalités pédagogiques et le déroulement de la formation : cohésion de groupe, pédagogie active, rencontres. Ces questions sont très intéressantes puisqu'elles renseignent sur l'ambiance de la formation et le bien-être de l'étudiant : outre les apprentissages, a-t-il passé une bonne semaine ? A-t-il rencontré des étudiants qu'il a appréciés ?

Les évaluations ont été réalisées en deux temps :

Au fil de la formation : questionnaire quotidien permettant d'évaluer chaque module (environ 50 répondants par jour). Le questionnaire est envoyé par mail chaque soir afin d'être complété par l'étudiant une fois de retour chez lui. Ce questionnaire est chaque jour différent puisqu'il interroge les étudiants sur l'atteinte des objectifs d'apprentissage qui sont fixés par chaque formateur, en coopération avec les équipes des deux IDEFI qui ont créé la semaine de formation. Toutefois, une partie du questionnaire reste la même et s'intéresse aux acquis de la formation: qu'ont-ils appris ? Que vont-ils faire des outils et techniques qui leur ont été transmis ? Comment vont-ils intégrer les acquis de la formation dans leurs études et dans leur vie personnelle?

Ces évaluations donnent lieu à un rapport d'évaluation quotidien réalisé par TalentCampus et transmis notamment au formateur. Cela vient alors compléter les éléments de débriefings recueillis à chaud le soir par le formateur et l'équipe de REMIS présente au quotidien pour encadrer la formation.

Après la semaine : questionnaire d'évaluation de l'ensemble de la formation (50 répondants/ 56 étudiants). Ce questionnaire s'intéresse aux objectifs d'apprentissage généraux de la semaine 
de formation mais également, tout comme les questionnaires quotidiens, aux acquis d'apprentissage et à la satisfaction générale. Ce rapport d'évaluation, combiné aux rapports quotidiens, permettent une analyse fine de ce qui s'est passé en formation et donnent la possibilité de faire évoluer la formation en vue d'une prochaine session.

Ces évaluations se font en ligne quotidiennement (s'agissant des évaluations de chaque journée) ou après la formation (ils ont alors un mois pour compléter l'évaluation de fin de formation), lorsque l'étudiant est de retour chez lui. Martens (2002) souligne en effet que s'il est demandé aux participants d'évaluer le programme directement à la fin de celui-ci, ces derniers sont généralement toujours satisfaits (Martens et coll., 2002), ce qui peut devenir un biais pour l'évaluation globale. La dernière journée de formation, décrite précédemment, est souvent une forme d'apothéose pour les étudiants qui sortent enthousiastes et «boostés »; une évaluation à chaud provoquerait de manière évidente un biais pour l'analyse qui en découlerait.

Les données recueillies par ces questionnaires auto-administrés ont été analysées par les évaluateurs selon une analyse de contenu thématique. Les réponses analysées ont ainsi été classées et ont permis de dégager différents thèmes et sous-thèmes que nous développerons dans l'analyse des résultats. Très souvent, nous observons que les étudiants sont nombreux à donner les mêmes réponses et cela permet d'obtenir des indications fiables sur ce que les étudiants retiennent et pensent de la formation.

Ce travail de conception de la formation et de l'évaluation résulte d'une coopération forte entre les équipes de TalentCampus et REMIS qui se sont appuyées sur l'expérience en matière d'innovation pédagogique de TalentCampus et se sont assurées de la bonne adéquation des objectifs d'apprentissage avec les activités proposées. Au sein de l'IDEFI REMIS, Pauline Thevenot, docteure en Sciences de l'Information et de la Communication depuis 2015, spécialiste de l'apprentissage par le jeu et de l'innovation pédagogique, est conceptrice scientifique. Elle intervient dans la mise en place de projets pédagogiques ludiques (serious games, ...) et co-organise des formations de formateurs ou d'étudiants pour expérimenter des formes de pédagogie atypique en rupture avec le système scolaire français classique. Raphaël Duval est professeur à l'Université de Lorraine depuis 2012. En 2015, il devient correspondant de l'IDEFI REMIS pour l'Université de Lorraine. La mise en place de la formation « Booster sa réussite » a été demandée et facilitée par ces deux personnes.

Au sein de l'IDEFI TalentCampus, Mélanie Souhait a porté le projet, organisé la formation avec l'université de Lorraine et coordonné l'évaluation de la formation. De plus, les réponses des étudiants étant hébergées sur une plateforme internet dédiée, TalentCampus a traité les 
réponses des étudiants de manière groupée afin d'obtenir un rapport d'évaluation sur l'ensemble de la formation. Il faut noter que les réponses des étudiants ne sont jamais traitées de manière individuelle afin de préserver leur anonymat et respecter leur liberté d'expression.

Le taux élevé de réponses ( $89 \%$ ) a permis d'avoir une bonne vue d'ensemble des effets de la formation.

\section{Résultats}

Plusieurs questions apparaissent lorsqu'on propose une formation de développement des compétences sociales. Les étudiants ont-ils réellement développé leurs compétences sociales? Ont-ils créé une cohésion de promotion ? Ont-ils acquis des techniques de travail en groupe et de communication interpersonnelle ? Quels bénéfices retirent-ils de cette semaine basée sur l'échange et le relationnel?

Répondre à ces interrogations est nécessaire puisque rappelons que les objectifs étaient de permettre à chacun de découvrir l'importance des compétences sociales, travailler l'estime de soi et développer des compétences de travail en groupe et de communication orale ; l'enjeu global étant, à travers des expérimentations loin des cours traditionnels proposés par l'enseignement universitaire, de rendre l'étudiant conscient de ses capacités, confiant en ses compétences et capable d'en parler et de les valoriser.

\subsection{Découverte des compétences sociales}

Naturellement, cette question est centrale puisqu'elle est au cœur de la formation. Or, $61 \%$ des répondants déclarent avoir découvert des compétences sociales et sont capables d'en citer. Ils sont $38 \%$ à répondre «non » à la question. Ces chiffres sont surprenants lorsqu'ils sont comparés aux taux habituels de réponses des formations animées par TalentCampus dans ou hors université, la découverte des compétences sociales étant souvent proche des $100 \%$. Cela peut s'expliquer par la diversité des étudiants (notamment diversité de parcours), mais aussi par le fait que les étudiants s'autocensurent devant leurs pairs, dans le cadre de cette formation plutôt personnelle. Ainsi, ils n'ont peut-être pas poussé le travail d'introspection autant qu'ils auraient pu pour réellement découvrir leurs compétences sociales. La découverte de soi est alors restée superficielle. Quelques réponses d'étudiants ayant répondu « oui » à la question montrent toutefois que les compétences sociales découvertes ou travaillées peuvent être variées :

«Je ne pensais pas être capable d'être aussi à l'aise en public en faisant parfois des choses un peu gênantes. » 
«La créativité face à des activités proposées. L'autonomie et l'apprentissage du travail de groupe. »

\subsection{Innovation de la formation}

$80 \%$ des répondants estiment cette formation comme étant innovante notamment car elle les a surpris par son côté ludique et décalé par rapport aux cours habituels. La rupture avec un apprentissage classique est essentielle car cela permet tout le travail réalisé autour de compétences non techniques que l'étudiant doit pouvoir expérimenter pour les développer. Il faut pourtant noter que les formations se sont déroulées au sein de l'université, dans des salles de cours. La rupture avec les habitudes des étudiants a donc bien été là. De plus, plusieurs étudiants soulignent les bénéfices du travail en groupe, notamment pour la découverte de soi et des autres. Ils notent également que les thèmes de la vie courante sont peu abordés (voire pas du tout) durant la scolarité et les études. Voici quelques illustrations collectées dans l'enquête : « La formation cible l'estime de soi, chose qui est très peu abordée. »

«La formation permet de se découvrir nous-même et les autres dans un contexte hors du commun, en s'amusant et en peu de temps. »

\subsection{Cohésion d'équipe}

Cette formation a eu de forts effets socialisants, en permettant de créer une cohésion de groupe ainsi que des relations nouvelles et durables. Ainsi, $100 \%$ des répondants estiment qu'il y a bien eu une cohésion de groupe durant la formation, ce qui ressort régulièrement dans les évaluations quotidiennes et dans l'évaluation finale.

«On n'a pas l'habitude de passer des semaines comme celles-ci, c'est fédérateur pour un groupe. »

« La formation permet de mieux connaître la promo dès le début de l'année en proposant des activités créatives. »

\subsection{Rencontres et relations durables}

$100 \%$ des répondants indiquent avoir rencontré des personnes qu'ils ne connaissaient pas. Pourtant, plusieurs étudiants se connaissaient déjà depuis la L1. Le fait d'être « obligés » de travailler avec de nouvelles personnes est bénéfique puisqu'il met les étudiants face à des 
simulations de futures expériences professionnelles coopératives. $94 \%$ des répondants estiment d'ailleurs avoir lié des relations durables (3/50 personnes ont répondu «non »).

«J'ai pu réellement travailler en équipe et gérer le temps avec des personnes que je ne connaissais pas. C'était très instructif et je me suis fait des amis. »

\subsection{Formation alternée grand groupe / petits groupes}

Tous les apprenants sont positifs quant à la méthode qui consiste à faire travailler les étudiants en groupes de 5-6 personnes au sein de la promotion de 56 étudiants. Cela contribue à la création d'un collectif. Par ailleurs, les groupes changent quotidiennement ce qui provoque les rencontres.

«C'est une méthode infaillible pour se connaître et connaître les autres, c'est une belle expérience qui mérite d'être vécue. »

«La formation est proche des conditions de travail que nous aurons plus tard, en essayant de varier à chaque fois les groupes pour travailler son adaptabilité. »

\subsection{Outils et techniques à réutiliser}

$80 \%$ des répondants citent des outils et des techniques assimilés pendant la formation et qu'ils pensent pouvoir réutiliser par la suite. Les principaux outils cités sont ceux de la gestion du stress (ancrage et respiration), mais aussi les techniques de mémorisation et celles de travail en groupe. Il est intéressant de noter que les étudiants sont capables d'indiquer comment et à quel moment ils seront capables de réutiliser ces techniques au cours de leur cursus universitaire. Citons par exemple :

« La carte mentale me servira pour préparer mes examens. »

«Faire une pause avec des activités manuelles (pendant une période de révision avant les partiels, par exemple) me permettrait de me vider la tête et de relâcher la pression. »

L'un des objectifs de la formation évaluée était de donner des clés aux étudiants pour mieux réussir leurs études. Au final, réussir à se projeter avec l'utilisation de nouveaux outils, doit donc être considéré comme un bénéfice que les étudiants retirent de cette formation. 


\subsection{Utilité des modules}

Le module le plus utile selon les étudiants est celui de début de semaine qui introduit le travail sur les compétences sociales et la découverte des mécanismes de travail en groupe. En plus d'avoir permis de créer une appartenance groupale, les étudiants ont pu développer des compétences plus «techniques » propres au travail en groupe : la gestion d'un projet collectif, la répartition des tâches, la gestion du temps et de la parole...

« Il m'a appris à mener un projet de groupe en se répartissant correctement les tâches, ce dont je n'ai pas l'habitude puisque je travaillais la plupart du temps seule. »

«J'ai pu réellement travailler en équipe et gérer le temps avec des personnes que je ne connaissais pas. C'était très instructif et je me suis fait des amis. »

À la question : «quel module vous a été le moins utile ?»50\% des apprenants ont indiqué le module relatif à la communication ; pourtant, l'année précédente ce module avait été largement plébiscité comme étant le module le plus utile. En 1 an, la technique d'enseignement de ce module a été modifiée, notamment l'alternance des présentations orales devant des groupes différents à chaque exposé. Lors la session ici présentée, les groupes sont restés fixes ce qui n’a pas permis aux étudiants de sortir de leur zone de confort et d'expérimenter la confrontation à un public inconnu. Au contraire, ils ont ressenti une certaine routine à présenter leurs textes toujours devant les mêmes personnes. D'ailleurs $43 \%$ des étudiants ont indiqué spontanément que le module avait été trop « répétitif ». Pour nous, cela signifie que la technique de formation employée doit donc rester en rupture avec l'enseignement classique (groupes aléatoires, remixés quotidiennement et régulièrement dans la journée, confrontations régulières avec l'inconnu pour se dépasser) comme cela s'est fait la première année et a contribué à rendre la formation utile. Car, nous constatons ici, que la forme d'enseignement lorsqu'elle n'est plus en rupture, ou s'inscrit dans une routine, réduit son efficacité en matière de développement des compétences sociales.

En outre, toujours à la question : «quel module vous a été le moins utile ? Pourquoi ? », trois modules n'ont jamais été cités : celui qui introduit la formation et donne des outils de travail en équipe (jour 1), celui qui pousse les étudiants à sortir de leur zone de confort en proposant des activités sportives (yoga, escalade et crossfit - jour 4) et le module de la dernière journée (jour 5) qui propose un récapitulatif de la semaine par les étudiants sous le format de leur choix (théâtre, chanson...). Notre interprétation de ces résultats est la suivante : le développement des compétences sociales est l'objectif de ces modules, mais les techniques d'apprentissage 
employées ne le révèlent pas directement. L'atelier n'est pas téléguidé. Il est comme un tas de sable. Chacun y puise ce dont il a besoin et l'utilise selon ses propres modalités. Selon nous, ces modules plus « ouverts » étaient les plus risqués en termes d'enseignement, car ce sont ceux qui offrent le plus de liberté et sont en rupture nette avec la forme universitaire classique. Nous ne pouvions pas être sûrs que les apprenants sauraient mettre en relation les notions « vécues » pendant ces modules, comme le dépassement de soi avec le sport ou le travail en groupe lors de la dernière journée - avec le développement de leurs compétences sociales. Pourtant, bien que ces modules ne soient pas forcément plébiscités parmi les modules les plus utiles (sauf celui sur le travail d'équipe) et grâce à la liberté qu'ils offrent dans l'apprentissage des compétences sociales, ces modules ont permis à $100 \%$ des apprenants d'y trouver une utilité). Les modules « tas de sable » sont donc précieux puisqu'ils sont utiles pour tous.

\subsection{Apports de la formation}

$75 \%$ des répondants à l'évaluation citent les nouvelles rencontres, le côté fédérateur au sein de la promotion, et de nouvelles amitiés comme bénéfices de la formation. Rien d'autre sur les apports de la formation?

«Je me sens maintenant très à l'aise dans ma promotion, et tous les matins je suis contente d'aller en cours. »

«Grâce à cette formation, j'ai rencontré de belles personnes et elle m'a permis aussi de voir qu'un groupe pouvait être bienveillant. »

\section{Discussion}

Nous évoquions en introduction l'importance de développer les compétences sociales des individus dans un objectif notamment de réussite scolaire mais aussi de bien-être (OCDE, 2015 ; Moscovici et coll., 2005). À la lecture des réponses des étudiants ayant participé à la formation, la question du bien-être est largement abordée. En effet, s'il est trop tôt pour dire que le travail sur les compétences sociales de ces étudiants leur permettra de mieux réussir dans leurs études, le fait de leur avoir permis de créer une réelle cohésion de classe est déjà très positif. De plus, une semaine de formation ne peut pas permettre de fortement développer des compétences sociales, mais peut servir de déclencheur, de prise de conscience de leur rôle dans la vie des étudiants, du point de vue de leur «métier » d'étudiant ou de leur vie personnelle. Un travail est amorcé et les étudiants, conscients de leurs capacités, vont pouvoir continuer à 
s'appuyer sur les acquis de la formation pour véritablement faire de ces compétences sociales une force dans leur parcours d'étudiant puis, à plus long terme, de collaborateur en entreprise. Par ailleurs, nous nous interrogions sur le fait que le dispositif ait bien permis aux étudiants de développer une cohésion de groupe et d'acquérir des outils et techniques de travail en groupe. Les témoignages des étudiants attestent des nombreux échanges entre camarades durant la formation et de leurs effets au niveau individuel et collectif. Les nombreuses activités collectives et coopératives qui leur ont été proposées ont été des outils efficaces permettant de favoriser la cohésion d'équipe, le développement de compétences et la co-construction de connaissances sur soi et sur les autres. Ils ont par ailleurs majoritairement pris conscience de l'importance et du rôle des compétences sociales tout au long de leur vie, mais également de l'importance de l'« Autre » dans l'évolution de ces compétences.

Toutefois, si les compétences sociales ont bien été travaillées, les étudiants n'en sont pas toujours conscients puisque nous avons vu que seuls $61 \%$ des étudiants déclarent avoir découvert des compétences sociales. Or, TalentCampus après plusieurs années d'expérience auprès de 35000 apprenants considère que chaque personne dispose de plusieurs compétences sociales fortes (appelées aussi talents), plus ou moins connues par l'individu et/ou son entourage, ou à découvrir. Il apparait que les problématiques sociales telles que l'anxiété sociale ou la timidité proviennent de l'inhibition des compétences sociales (Nader-Grosbois, 2007 ; George, 2001). Elles seraient bien apprises par le sujet et donc présentes, mais étant inhibées par certaines situations sociales, elles ne peuvent être utilisées (George, 2001). Le lien entre la formation proposée et le développement des compétences sociales est-il clair pour les étudiants? Les formateurs devraient davantage contextualiser la formation. Il est en effet essentiel d'annoncer les objectifs d'apprentissage en début de journée, puis faire le lien entre les activités proposées et ces objectifs à travers des débriefings pédagogiques tout au long de la journée. Pour réussir cela, il est nécessaire de davantage accompagner les formateurs dans leur manière de penser et mettre en place leur formation.

Il serait également intéressant de proposer ce type de formation à plusieurs temps de l'année universitaire afin de maintenir le travail coopératif engagé, mais aussi pour poursuivre et compléter le travail amorcé sur les compétences sociales. Ce travail doit se faire de manière régulière et continue, et selon les besoins des étudiants qui évoluent constamment. Les bénéfices de cette première intervention soulignent en effet la nécessité de travailler et de développer les compétences sociales des étudiants pour les aider sur plusieurs aspects : faire face à la transition entre les différentes filières et les différents milieux de formation, s'insérer 
dans le milieu professionnel plus facilement (être plus « attractifs » pour leurs employeurs) et être plus performants au travail (dans les études et dans le monde professionnel).

Au niveau de l'évaluation, une approche combinant un pré et post test et une comparaison à un groupe témoin semble la plus efficace et la plus complète pour mesurer l'évolution des changements de compétences sociales. Par ailleurs, il est nécessaire que le groupe témoin dispose de caractéristiques similaires au groupe qui reçoit le programme pour une meilleure fiabilité des résultats. Nous notons également que de nombreux chercheurs préconisent une approche de «méthodes mixtes» combinant des approches à la fois quantitatives et qualitatives, dans l'objectif de complexifier le processus analytique et ainsi, de renforcer la validité des conclusions (Blouin, 2013). Cette perspective méthodologique permettrait en effet de mieux saisir la complexité de la notion de compétences sociales, et de son évolution.

\section{Conclusion}

Au regard des évaluations quotidiennes et de l'évaluation finale, il apparaît que les étudiants sont très positifs quant à leur semaine de formation. Les méthodes pédagogiques proposées, ludiques et actives, les ont surpris et ont permis les échanges nombreux. Ainsi, beaucoup expliquent que la semaine de formation leur a permis de créer une cohésion de promotion forte, de parler avec de nombreuses personnes pour la toute première fois, et de créer des liens, voire des amitiés entre étudiants.

Certaines activités proposées, comme lors de la journée de communication le $4^{\mathrm{e}}$ jour sont à revoir car les étudiants sont nombreux à ne pas avoir adhéré et expliquent que les activités étaient trop statiques et manquaient de dynamisme. Cela signifie bien que les étudiants ont adopté la pédagogie active très vite et ont finalement été surpris par cette journée plus classique. Par ailleurs, nous avons vu que davantage d'explications de la part du formateur sur le lien entre les activités chaque jour et leur enjeu pédagogique permettraient aux étudiants de mieux comprendre les objectifs de la formation. Ainsi, les étudiants seraient davantage en capacité d'expliquer ce qu'ils ont appris car le travail sur des compétences sociales, donc non « tangibles », peut-être difficile à cerner : quel est mon niveau d'estime de soi ? Quelles sont vraiment mes compétences sociales fortes? Suis-je bon communiquant? Ces interrogations que l'on amène les étudiants à se poser durant la formation ne sont pas simples, surtout lorsque c'est la première fois que l'on y réfléchit. 
Cette volonté de faire progresser la formation qui a émergé suite à cette $2^{\mathrm{e}}$ expérimentation (la toute première session de formation «Booster sa réussite » s'était déroulée en septembre 2017) et le besoin d'améliorer encore cette semaine de formation ont une nouvelle fois suscité l'envie, au sein des deux IDEFI, de proposer à chaque rentrée cette formation unique au sein de l'Université de Lorraine. En 2019, TalentCampus souhaitait proposer une formation de formateurs aux membres de l'équipe pédagogique de la L2 Sciences pour la Santé. Cela aurait permis de transmettre la formation afin de proposer chaque année une semaine particulièrement appréciée des étudiants, car permettant d'apprendre de manière nouvelle, ludique et coopérative. Cela n'a pas encore été mis en place à l'Université de Lorraine mais cette démarche d'essaimage a été initiée depuis plusieurs années par TalentCampus auprès d'autres universités en France et à l'étranger. Il est clair que l'importance des compétences sociales dans une vie d'étudiant (puis dans la vie professionnelle) est une réalité désormais reconnue dans le monde universitaire aujourd'hui.

\section{Références bibliographiques}

Abbet, J.-P. \& Moreau, J. (2012). Les compétences sociales et leur place dans la formation : résultats d'une enquête auprès des élèves, apprentis et gymnasiens vaudois, ainsi que de leurs enseignants. URSP, Unité de Recherche pour le Pilotage des Systèmes Pédagogiques.

Baron, R. A. \& Markman, G. D. (2000). Beyond social capital: How social skills can enhance entrepreneurs' success. The Academy of Management Executive, 14(1), 106-116.

Barth, B.M. (1993). Le savoir en construction, former à une pédagogie de la compréhension, Paris : Retz.

Biggs, J. (2003). Aligning teaching and assessing to course objectives. Teaching and Learning in Higher Education: New Trends and Innovations. University of Aveiro, 13-17 April, 2003.

Blouin, G. (2013). «L'utilisation des méthodes mixtes pour appréhender la complexité des enjeux de santé publique », Conférence "Santé et sociétés : Réflexions sur l'utilisation des méthodes mixtes en recherche », Rennes (France), November 20th.

Charlot, B., Bautier, E. \& Rochex, J.-Y. (1992). École et savoir dans les banlieues et ailleurs. Paris : Armand Colin.

Coulet, J.-C. 2011. La notion de compétence : un modèle pour décrire, évaluer et développer les compétences. Le travail humain, 74(1), 1-30.

Curchod, P., Doudin, P.-A., Lafortune, L. \& Collectif. (2012). Les transitions à l'école. Québec, Presses de 1'Université du Québec.

Desbiens, N., Royer, E., Bertrand, R. \& Fortin, L. (2000). La réputation sociale des élèves en difficulté de comportement : Impact d'un programme d'entraînement aux habiletés sociales et de coopération en classe ordinaire. Revue Québécoise de Psychologie, 21(2), 57-79. 
Drozda-Senkowska, E., Gasparini, R., Huguet, P., Rayou, P. \& Filisetti, L. (2002). Acquisition et régulation des compétences sociales. Paris : Ministère de la Recherche.

Durlak, J. A., Weissberg, R. P., Dymnicki, A. B., Taylor, R. D. \& Schellinger, K. B. (2011). The impact of enhancing students' social and emotional learning: A meta-analysis of schoolbased universal interventions. Child Development, 82, 405-432.

Duru-Bellat, M. (2004). La ségrégation sociale à l'école : Faits et effets. VEI, 139, 73-78.

Eneau, J. \& Simonian, S. (2011). Un scénario collaboratif pour développer l'apprentissage d'adultes, en ligne et à distance. Recherche \& formation, 68(3), 95-108.

George, G. (2001). Les thérapies d'affirmation de soi : applications et techniques chez les adolescents. Annales Médico-psychologiques, revue psychiatrique, 159(10), 717-721.

Giret, J. F. \& Morlaix S. (2016). Les compétences sociales et non académiques dans les parcours scolaires et professionnels. Éditions Universitaires de Dijon, 158 p..

Goleman, D. (1999). L'intelligence émotionnelle : maîtriser nos émotions pour améliorer nos compétences personnelles et sociales. Paris : Robert Laffont.

Hernandez, L. (2016). Programmes de développement des compétences sociales et d'insertion professionnelle chez les étudiant.e.s : efficacité et limites, L'orientation scolaire et professionnelle, 45(3), p.303.

Joannaert, P. (2009). Compétences et socioconstructivisme : un cadre théorique. Bruxelles : De Boeck.

Kolb, D. A. (1984). Experiential learning: experience as the source of learning and development. Prentice Hall : Englewood Cliffs. Traduction de Samuel Chartier.

Martens, H., Grieten, S., De Weerdt, S. \& Schippers, N. (2002). Experiences with training in social skills: design and study of the effectiveness: a case study at the Faculty of Management of the Limburg University Centre. Argumenta Oeconomica, 2(13), 149-172.

Moscovici, S., Argyle, M., Beauvois, J.-L. \& Doise, W. (2005). Psychologie sociale des relations à autrui. Paris : Armand Colin.

Nader-Grosbois, N. (2007). Régulation, autorégulation, dysrégulation : pistes pour l'intervention et la recherche. Wavre (Belgique) : Mardaga.

Nangle, D. W., Hansen, D. J., Erdley, C. A., Norton, P. J. (Eds.) 2010. Practitioner's guide to empirically based measures of social skills. New York: Springer.

OCDE. (2015). Skillsfor Social Progress: The Power of Social and Emotional Skills. Paris: Organisation for Economic Co-operation and Development.

Sarrazin, P., Tessier, D. \& Trouilloud, D. (2006). Climat motivationnel instauré par l'enseignant et implication des élèves en classe : l'état des recherches. Revue Française de Pédagogie, 157, 147-17.

Segrin, C. (2000). Social skills deficits associated with depression. Clinical Psychology Review, 20, 379-403. 
Spence, S. H. (2003). Social skills training with children and young people: theory, evidence and practice. Child and Adolescent Mental Health, 8(2), 84-96.

Vygotsky, L. S. (1978). Mind in society: The development of higher psychological processes. Cambridge, MA : Harvard University Press.

Wallon, H. (1941). L'évolution psychologique de l'enfant. Paris : Armand Colin. 